\title{
Autopsie: Ersetzt das CT den Pathologen?
}

\begin{abstract}
Nicht nur die konventionelle medizinische Autopsie, auch die postmortale Computertomografie bestätigt die Mehrzahl der klinischen Diagnosen und entdeckt unbekannt gebliebene Erkrankungen.
\end{abstract}

- Die Studie erfolgte auf den neun Intensivstationen der Universitätsklinik Hamburg-Eppendorf. Innerhalb von sechs Monaten starben 285 Patienten. In 47 Fällen erlaubten die Angehörigen sowohl eine konventionelle Autopsie als auch eine postmortale Computertomografie (virtuelle Autopsie).

Von den 196 klinischen Diagnosen zu Lebzeiten konnten 173 (88\%) durch die virtuelle, 183 (93\%) durch die konventionelle Autopsie bestätigt werden.

Es gab 14 neue, klinisch bedeutsame Diagnosen: Zehn wurden durch beide Methoden entdeckt, vier nur durch die konventionelle Autopsie, nämlich zwei Lungenembolien, eine alveoläre Proteinose und eine septische Kniegelenksarthritis (außerhalb des CT-Areals). Von

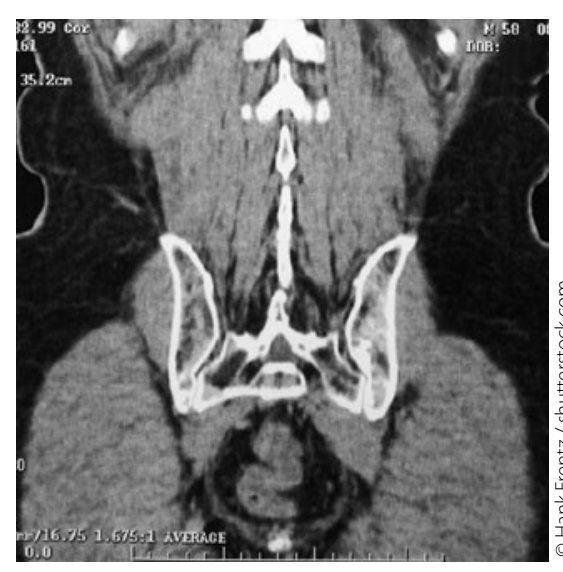

Ein CT deckt Diagnosen auf, die selbst der Autopsie entgehen.

88 weiteren Nebendiagnosen wurden 26 durch beide Methoden, 27 nur durch virtuelle und 35 nur durch konventionelle Autopsie entdeckt. Bei der Computertomografie werden vor allem Karzinome, Thrombosen, Lungenembolien und Myokardinfarkte, bei der konventionellen Autopsie vor allem Frakturen, Pleura- und Perikardergüsse, Pneumothoraces und Fremdkörper übersehen.

\section{Kommentar}

Zusammenfassend werden durch beide Methoden $88 \%$ aller klinischen Diagnosen bestätigt und eine nennenswerte Zahl neuer Diagnosen entdeckt. Die virtuelle Autopsie bestätigt nicht alle Diagnosen, entdeckt aber auch bislang unbekannte Erkrankungen. Sie eignet sich besonders gut für Todesfälle durch Trauma oder Schussverletzungen. Andererseits schwächelt das CT bei kardiovaskulären Komplikationen wie Herzinfarkt und KHK und - anders als bei Patienten mit intaktem Kreislauf-bei Lungenembolien. Man könnte Computertomografen in der Pathologie installieren, um virtuelle und konventionelle Autopsie durch Kombination zu optimieren und um das CT als Alternative zu haben, wenn Angehörige eine medizinische Autopsie ablehnen. Kliniken ohne eigene Pathologie könnten auf die virtuelle Autopsie ausweichen.

H. HolzGReVE =

\section{- D. Wichmann et al.}

Virtual autopsy as an alternative to traditional medical autopsy in the intensive care unit. Ann. Intern. Med. 156 (2012) 123-130

\section{Mehr Schlaganfälle bei depressiven Patienten}

\section{Depressionen wurden mit einem erhöhten Risiko für kardiovaskuläre Erkrankungen in Zusammenhang ge- bracht. Eine Metaanalyse belegt nun, dass auch ein erhöhtes Schlaganfall- risiko besteht.}

— Für die Metaanalyse standen Daten von 317540 Studienteilnehmern zur Verfügung. Depressionen zu Studienbeginn wurden mit Symptom-Fragebögen oder durch klinische Diagnosen erfasst. Zur Bestimmung von Schlaganfällen im Verlauf wurden Krankenakten, Totenscheine und Selbstangaben der Studienteilnehmer herangezogen.

Während des zwei bis 29 Jahre dauernden Follow-up waren 8478 Schlaganfälle aufgetreten. Personen mit einer Depres- sion zu Studienbeginn hatten ein um 45\% erhöhtes Schlaganfallrisiko im Vergleich zu Personen ohne eine Depression.

\section{Kommentar}

Die genauen Mechanismen dieses Zusammenhangs zwischen Depression und Schlaganfall sowie anderen kardiovaskulären Erkrankungen bedürfen der weiteren wissenschaftlichen Klärung. Auf pathobiologischer Ebene ist denkbar, dass Depressionen über bereits bekannte neuroendokrine, proinflammatorische oder prothrombotische Effekte zu einer Erhöhung des vaskulären Risikos führen. Darüber hinaus ist bekannt, dass Depressionen mit ungesunden Lebensstilfaktoren wie Rauchen und Bewegungsmangel sowie auch mit einer verminderten Medikamentenadhärenz in der Therapie von kardiometabolischen Risikofaktoren einhergehen.

Unabhängig von den auslösenden Mechanismen kann empfohlen werden, dass Patienten mit Depression besonders aufmerksam auf modifizierbare Risikofaktoren untersucht und entsprechend behandelt bzw. zur Verhaltensprävention motiviert werden. Umgekehrt sollten bei Patienten mit erhöhtem vaskulären Risiko depressive Symptome gezielt erfragt und in der Risikoabschätzung mit berücksichtigt werden.

M. BUSCH =

\footnotetext{
- A. Pan et al.

Depression and risk of stroke morbidity and mortality: A meta-analysis and systematic review. JAMA 306 (2011) 1241-1249
} 